\title{
Insuficiência cardíaca e atividade física: 0 aumento de enzimas antioxidantes e a melhora da atividade nervosa simpática
}

\author{
Heart failure and physical activity: the increase in antioxidant enzymes and improvement \\ of sympathetic nerve activity
}

Rodrigo Augusto dos Santos Gonçalves' ${ }^{1}$ Vitor Engrácia Valenti ${ }^{1}, 2$, Celso Ferreira ${ }^{3}$, Neif Murad ${ }^{3}$, Luiz Carlos de Abreu'

\section{Resumo}

A insuficiência cardíaca vem se tornando um problema cada vez maior em saúde pública, e uma das principais causas de internações hospitalares em todo o mundo. Mesmo com os progressos médicos e os avanços tecnológicos atuais, a prevalência da doença aumentou nas últimas cinco décadas. Estudos recentes com animais mostraram que existe uma relação entre hiperatividade simpática na insuficiência cardíaca e aumento do estresse oxidativo sistêmico e central. Este trabalho tem como objetivo analisar, por meio de revisão bibliográfica, os benefícios produzidos pela atividade física em pacientes portadores de insuficiência cardíaca. Foram revisados estudos relacionados ao estresse oxidativo, controle neural da circulação, insuficiência cardíaca e exercício físico entre outubro de 2009 e abril de 2010 nas bases de dados: Medline, Pubmed e Lilacs. As referências eram datadas de 1986 a 2010. Utilizaram-se os seguintes termos: "Oxidative Stress", “Chronic Heart Failure", "Baroreflex", “Nervous System" and "Exercise". Ratos com insuficiência cardíaca induzida possuem um aumento de espécies reativas de oxigênio na região rostroventrolateral do bulbo, assim como comprometimento na atividade simpática. Alguns estudos sugerem que a atividade física normaliza a atividade simpática por meio de seus mecanismos antioxidantes em seres humanos. Outro aspecto interessante é que a atividade física reduz os níveis plasmáticos de angiotensina ll, regula a disfunção do baroreflexo arterial e promove o aumento de enzimas antioxidantes no músculo esquelético de pacientes com insuficiência cardíaca. Portanto, conclui-se que a atividade física é um regime terapêutico não-farmacológico de extrema importância para pacientes com insuficiência cardíaca.

Palavras-chave: Insuficiência cardíaca; estresse oxidativo; exercício físico; sistema neural.

\begin{abstract}
Heart failure is an increasing problem in public health, and a major cause of hospitalization worldwide. Even with the medical progress and technological advances in the present, the prevalence of the disease increased in the last five decades. Recent animal studies have shown that there is a relationship between sympathetic hyperactivity in heart failure and increased oxidative stress, systemic and central. This paper aims to examine, through literature review, the benefits of physical activity in patients with heart failure. We reviewed studies related to oxidative stress, neural control of circulation, heart failure and exercise between 0ctober 2009 and April 2010, on the databases: Medline, Pubmed e Lilacs. The references dated from 1986 to 2010, and we used the following terms: "oxidative stress", "chronic heart failure", "baroreflex", "nervous system" and "exercise". Rats with induced heart failure have an increase of reactive oxygen species in the rostroventrolateral medula, and impairment of sympathetic activity. Some studies suggest that physical activity normalizes the sympathetic activity through its antioxidant mechanisms in the rostroventrolateral medulla. Some studies suggest that physical activity normalizes sympathetic activity through its antioxidant mechanisms in humans. Another interesting aspect is that physical activity reduces plasma levels of angiotensin II, regulates the baroreflex dysfunction of the blood and promotes the increase of antioxidant enzymes in skeletal muscle of patients with heart failure. We concluded that physical activity is anm extremely important non-pharmacological treatment for patients with heart failure.
\end{abstract}

Keywords: Heart failure; oxidative stress; exercise; nervous system.

Recebido: $7 / 3 / 2010$

Revisado: $10 / 6 / 2010$

Aprovado: $23 / 8 / 2010$

\footnotetext{
Trabalho realizado na Faculdade de Medicina do ABC (FMABC), Santo André, (SP) Brasil

Departamento de Morfologia e Fisiologia, Disciplina de Fisiologia da Faculdade de Medicina do ABC (FMABC), Santo André, (SP) Brasil

2 Departamento de Medicina, Disciplina de Cardiologia da Universidade Federal de São Paulo (UNIFESP), São Paulo, (SP) Brasil.

${ }^{3}$ Departamento de Clínica Médica, Disciplina de Cardiologia da Faculdade de Medicina do ABC (FMABC), Santo André, (SP) Brasil

Endereço para correspondência: Rodrigo Augusto dos Santos Gonçalves - Faculdade de Medicina do ABC, Departamento de Fisiologia - Av. Lauro Gomes

2000 - Vila Sacadura Cabral - CEP 09060-870 - Santo André (SP), Brasil - e-mail: rodrigoaugustoo@gmail.com
} 


\section{Introdução}

A Insuficiência Cardíaca (IC) vem se tornando um problema cada vez maior em saúde pública, e uma das principais causas de internações hospitalares em todo o mundo, gerando, assim, um grande impacto econômico na sociedade ${ }^{1}$. Mesmo com os progressos médicos e os avanços tecnológicos atuais, a prevalência da doença aumentou nas últimas cinco décadas e, ainda hoje, a mortalidade pode ultrapassar $50 \%$ em cinco anos, a partir do momento do seu diagnóstico ${ }^{2,3}$. Dados epidemiológicos revelam que a incidência de IC após os 65 anos de idade aproxima-se de dez casos em 1.000 indivíduos, e apesar dos avanços no tratamento, 300 mil pacientes morrem por ano ${ }^{4,5}$. No Brasil, segundo o Sistema Único de Saúde (SUS), foram realizadas, no ano de 2000, aproximadamente 398 mil internações por IC com aproximadamente 26 mil óbitos, cerca de um terço dos internados no SUS com doenças cardíacas é portador de $\mathrm{IC}^{6}$. Estudos neuroquímicos e neurofisiológicos revelam que um dos fatores que levam ao agravamento da IC crônica é o aumento da atividade nervosa simpática ${ }^{7-9}$. Essa anormalidade simpática é um ajuste compensatório para promover adequada perfusão tecidual periférica que, com o passar do tempo, torna-se cada vez mais intensa, contribuindo para a progressão da insuficiência cardíaca ${ }^{10}$. Grandes evidências sugerem que a região rostroventrolateral do bulbo (RVL), uma importante região do sistema nervoso central responsável por excitar tonicamente neurônios simpáticos pré-ganglionares localizados na coluna intermédio-lateral regulando a atividade simpática para o sistema cardiovascular, seja modulada por espécies reativas de oxigênio, aumentando a transmissão simpática e, portanto, corroborando para a piora do estado clínico em indivíduos com $\mathrm{IC}^{11-16}$. Acredita-se que um aumento na atividade nervosa simpática possua um importante papel no desenvolvimento de algumas doenças cardiovasculares como, por exemplo, insuficiência cardíaca e hipertensão arterial ${ }^{17,18}$.
Alguns estudos têm mostrado que atividade física tem se tornado uma estratégia terapêutica não-farmacológica em indivíduos com insuficiência cardíaca crônica, promovendo uma melhora no estado oxidativo e redução da atividade nervosa simpática, dentre outros ${ }^{10,19,20}$. Além disso, estudos recentes em animais mostraram que existe relação entre hiperatividade simpática na insuficiência cardíaca e aumento do estresse oxidativo sistêmico e central ${ }^{10,11}$.

Em vista dos fatores acima apresentados, nosso objetivo é descrever os efeitos e os benefícios da atividade física em indivíduos com insuficiência cardíaca crônica.

\section{Método}

Foram revisados estudos relacionados ao estresse oxidativo, controle neural da circulação, insuficiência cardíaca e exercício físico entre outubro de 2009 e abril de 2010, nas bases de dados: Medline, Pubmed e Lilacs. As referências eram datadas entre os anos de 1986 e 2010. A busca teve como base as combinações das palavras "oxidative stress", "chronic heart failure", "baroreflex", "nervous system” e "exercise”. Após a seleção de todos os artigos cabíveis para a revisão, o número total de artigos utilizados foi 67. As referências dos respectivos artigos foram examinadas.

\section{Resultados}

Os benefícios da atividade física estão relacionados ao seu efeito antioxidante, os quais não são produzidos somente por um aumento na expressão de enzimas antioxidantes, mas também por uma redução na expressão de enzimas pró-oxidantes. Conforme apresentado na Tabela I, alguns estudos sugerem que a atividade física normaliza a atividade simpática por meio de seus mecanismos antioxidantes na região RVL, e

Tabela I - Benefícios produzidos pela atividade física.

\begin{tabular}{|c|c|}
\hline Autor & Aspecto estudado \\
\hline \multirow[b]{2}{*}{ Lie Gao et al..$^{10}$} & $\begin{array}{l}\text { Expressão proteica de superóxido dismutase e subunidade da } \\
\text { NAD(P)H oxidase na RVL. }\end{array}$ \\
\hline & $\begin{array}{l}\text { Após a realização de Western Blot, verificaram uma diminuição } \\
\text { na expressão proteica de CuZnSOD/ MnSOD,e, aumento da } \\
\text { subunidade da enzima NAD(P)H oxidase, gp91 phox. }\end{array}$ \\
\hline \multirow[t]{2}{*}{ Axel Linke et $a / .^{47}$} & $\begin{array}{l}\text { Avaliação da atividade enzimática da Catalase, Superóxido Dis- } \\
\text { mutase e Glutationa Peroxidase no músculo esquelético através } \\
\text { de biópsia. }\end{array}$ \\
\hline & $\begin{array}{l}\text { Encontraram que a atividade dessas enzimas estava diminuída no } \\
\text { músculo esquelético de indivíduos com insuficiência cardíaca. }\end{array}$ \\
\hline & Níveis de Angiotensina II no plasma. \\
\hline Jun- Li Liu et $a{ }^{61}$ & $\begin{array}{l}\text { Coelhos com insuficiência cardíaca que não realizaram atividade } \\
\text { física possuíam um aumento significatico de angiotensina II no } \\
\text { plasma. }\end{array}$ \\
\hline
\end{tabular}

Expressão das subunidades da enzima $\mathrm{NAD}(\mathrm{P}) \mathrm{H}$ oxidase e de Volker Adams et al. ${ }^{20}$ receptores AT1-R e AT2-R em pacientes com doença arterial coronariana.

Sensibilidade do Baroreflexo.

Bertagnolli et al. ${ }^{37} \quad$ As respostas do baroreflexo, a bradicardia e taquicardia estavam diminuídas em SHR sedentários
Resultados da atividade física

A atividade física aumentou a regulação da expressão proteica dessas enzimas e diminui a expressão proteica de gp91phox na RVL.

Após seis meses de realização de atividade física houve um aumento na atividade da GPX e CAT no músculo esquelético. Não houve alterações significantes na SOD.

Coelhos com insuficiência cardíaca que realizaram atividade física exibiram uma diminuição na angiotensina II no plasma.

A atividade física diminuiu a expressão para mRNA da subunidade da NAD(P)H oxidase, gp91 phox. Promoveu também a redução da expressão para mRNA dos receptores AT1- $R$, e, a expressão para os receptores AT2-R estava aumentada após o treino.

A atividade física restabeleceu as respostas do baroreflexo a bradicardia e taquicardia em SHR treinados. 
aumenta a sensibilidade do baroreflexo arterial, que está diminuída no estado de insuficiência cardíaca. A atividade física também promove efeitos antioxidantes no músculo esquelético de pacientes com insuficiência cardíaca, por promover um aumento da atividade de enzimas varredoras de radicais, reduzindo o dano às fibras musculares e evitando a perda de massa muscular.

Outro dado interessante é que a atividade física reduziu a concentração plasmática de angiotensina II, diminuiu a expressão dos receptores AT1 e aumentou a expressão dos receptores AT2 (Tabela I). Em virtude disso, a atividade física tem se mostrado como uma estratégica terapêutica não-farmacológica na prevenção e reabilitação de muitas doenças cardiovasculares.

\section{Discussão}

Espécies reativas de oxigênio são moléculas que contêm oxigênio em um estado altamente reativo, e são encontradas em todos os sistemas biológicos podendo atuar como moléculas sinalizadoras para regular a função biológica ${ }^{21-24}$. Estudos demonstraram que o peróxido de hidrogênio, que também é uma espécie reativa de oxigênio, atua no sistema nervoso central modulando a transmissão sináptica, e ativa o sistema simpático e parassimpático produzindo respostas pressoras e bradicardia ${ }^{25-27}$.

A IC é uma síndrome clínica complexa, definida como incapacidade do coração em bombear sangue, em relação ao retorno venoso e às necessidades metabólicas teciduais, ocasionando redução do débito cardíaco e elevação das pressões pulmonares e venosa sistêmica. Quando ocorre queda da função cardíaca, mecanismos adaptativos, como aumento da atividade simpática, são estimulados para corrigir a disfunção ventricular $28,29,7-9$.

Esse aumento da atividade simpática é um ajuste compensatório a uma redução na função cardíaca que, com o passar do tempo, torna-se cada vez mais intenso e sustentado, promovendo vasoconstrição, retenção de sódio, e contribuindo para um declínio progressivo da função contrátil do miocárdio, facilitando o desenvolvimento de arritmias ventriculares e promovendo efeitos nocivos da norepinefrina sobre os cardiomiócitos ${ }^{9,30,19}$.

Indivíduos portadores de IC estão sujeitos ao aumento do estresse oxidativo, e estudos anteriores revelam que o mesmo ao nível central, desenvolve um papel importante na descarga simpática no estado de insuficiência cardíaca. Lie Gao et al., em um estudo que analisou os efeitos da atividade física sobre a atividade simpática e o estresse oxidativo ao nível central em um modelo experimental de insuficiência cardíaca, encontraram que a mesma aumentou a expressão para CuZnSOD (cobre/zinco superóxido dismutase) e MnSOD (manganês superóxido dismutase) e diminuiu a expressão da subunidade da enzima NAD(P)H oxidase, gp91phox, na região RVL, normalizando a atividade simpática e melhorando a disfunção do baroreflexo arterial ${ }^{31,32,10}$.

O baroreflexo arterial é um importante regulador da pressão sanguínea, e sua sensibilidade é clinicamente relevante como um preditor de mortalidade cardiovascular. Alguns estudos mostram que a sua sensibilidade está reduzida na insuficiência cardíaca e no infarto agudo do miocárdio ${ }^{33,34}$

Algumas evidências sugerem que a atividade física de moderada a alta intensidade reduzem a pressão arterial e aumentam a sensibilidade do baroreflexo em ratos espontaneamente hipertensos (ratos esponataneamente hipertensos - SHR). Bertagnolli et al., em um estudo com SHR, postularam que o estresse oxidativo pode contribuir para uma disfunção no baroreflexo, e que a atividade física através de seus mecanismos antioxidantes pode melhorar a sua sensibilidade do mesmo ${ }^{35-37}$.

Outro dado importante sobre as espécies reativas de oxigênio, é que elas induzem a expressão de citocinas inflamatórias no músculo esquelético de pacientes com IC, e essas podem promover a produção de mais espécies reativas de oxigênio, levando, portanto, a um ciclo vicioso que causa o enfraquecimento da função muscular, apoptose das fibras musculares e consequente perda de massa muscular ${ }^{38-46}$.

Axel Linke et al., realizando um estudo para verificar a atividade de enzimas varredoras de radicais livres no músculo esquelético de indivíduos com IC, encontraram que a atividade física exerce efeitos anti-inflamatórios e anti-oxidantes em indivíduos com IC, diminuindo a expressão de citocinas inflamatórias e aumentando a atividade de enzimas varredoras de radicais, o que leva a uma clara atenuação do estresse oxidativo e a uma redução do dano aos miócitos do músculo esquelético causado por apoptose ${ }^{47}$.

Ennezat et al., realizando um experimento para determinar as bases moleculares da melhora na função endotelial de pacientes com IC submetidos a atividade física, encontraram que a mesma promoveu um aumento significante na expressão de genes que codificam as enzimas antioxidantes CuZnSOD e GPX (glutationa peroxidase), e postularam que uma redução no estresse oxidativo pode favorecer a vasodilatação mediada pelo óxido nítrico ${ }^{48}$.

Muitas substâncias humorais estão elevadas na IC, das quais podemos citar a angiotensina II, conhecida há algum tempo por modular a descarga simpática, pois quando se efetua bloqueio dos receptores AT1 para a angiotensina II, percebe-se uma redução do tônus simpático no estado de IC ${ }^{49-54}$.

Experimentos in vivo e in vitro, têm mostrado que a angiotensina II aumenta a formação de espécies reativas de oxigênio e a regulação da expressão proteica e de mRNA para subunidades de NAD(P)H oxidase, contribuindo, portanto, para a piora do estado oxidativo em indivíduos com insuficiência cardíaca ${ }^{55,56}$.

Lie Gao et al., em um estudo para analisarem o papel da angiotensina II e da $\mathrm{NAD}(\mathrm{P}) \mathrm{H}$ oxidase na IC, encontram que existe um aumento, na região RVL, da expressão proteica e de mRNA para os receptores AT1, e as subunidades da enzima NAD(P)H oxidase também estavam aumentadas na região RVL de coelhos com IC, e postularam que a angiotensina II através de seu receptor AT1, ativa mecanismos de descarga simpática por estimulação da $\mathrm{NAD}(\mathrm{P}) \mathrm{H}$ oxidase e espécies reativas de oxigênio, estabelecendo uma forte relação entre angiotensina 
II e espécies reativas de oxigênio como contribuidoras para a excitação simpática ${ }^{11}$.

Portanto, apesar de a angiotensina II exercer mecanismos fisiológicos clássicos, como vasoconstrição, retenção de sódio e água, dentre outros, e ser uma importante substância para a manutenção da homeostase cardiovascular, a literatura tem revelado uma íntima ligação entre angiotensina II e estresse oxidativo.

A elevação no estresse oxidativo induzido pela angiotensina II é contrabalanceada pela ativação dos receptores do tipo II (AT2-R), e a rede de efeitos da angiotensina sobre os leitos vasculares depende sobretudo da relação local entre os receptores AT1-R e AT2-R sobre a superfície celular ${ }^{57}$.

Em um estudo com pacientes com doença arterial coronariana realizado por Volker Adams et al., para analisarem o impacto da atividade física sobre o sistema de receptores da angiotensina e a atividade enzimática da $\mathrm{NAD}(\mathrm{P}) \mathrm{H}$ oxidase, verificaram que a atividade física reduziu a expressão das subunidades do complexo enzimático NAD(P)H oxidase, resultando em uma diminuição da produção total de ânions superóxido. Outro aspecto interessante neste estudo foi a diminuição para mRNA dos receptores AT1 e a expressão de mRNA para os receptores AT2 estava significativamente alta após o treino, resultando em uma diminuição na relação entre os receptores $\mathrm{AT} 1 / \mathrm{AT}^{20}$.
A literatura tem fornecido informações claras de que existe um aumento na concentração de angiotensina II no plasma na IC severa (classes 3 e 4 segundo a classificação da New York Heart Association), e evidências mostram que a mesma promove um efeito inibidor sobre a função do baroreflexo arterial ${ }^{58-60}$.

Jun-Li Liu et al., em um estudo para analisarem os efeitos da atividade física em um modelo experimental de IC, encontraram que a atividade física promoveu uma diminuição no estado excitatório simpático, bem como na concentração plasmática de angiotensina II, e sugeriram que a diminuição nos níveis de angiotensina II podem contribuir para o decréscimo da atividade nervosa simpática após a atividade física na insuficiência cardíaca ${ }^{61}$.

Em virtude dos fatos acima mencionados, pode-se concluir que a atividade física é benéfica para pacientes com insuficiência cardíaca crônica, pois a mesma diminui a atividade nervosa simpática, regula a sensibilidade do baroreflexo arterial, promove o aumento de enzimas antioxidantes e reduz os níveis plasmáticos de angiotensina II. Essa afirmação é sustentada por trabalhos bem aceitos pela literatura ${ }^{62-64}$. Além disso, indivíduos comprometidos com um protocolo de atividade física possuem um aumento da tolerância ao exercício, melhora na qualidade de vida, bem como aumento da sobrevivência ${ }^{65-67}$.

\section{Referências}

1. Cleland JG, Gemmell I, Khand A, Boddy A. Is the prognosis of heart failure improving? Eur J Heart Fail. 1999;1(3):229-41.

2. Anderson B, Waagstein F. Spectrum and outcome of congestive heart failure in a hospitalized population. Am Heart J. 1993;126(3 Pt 1):632-40.

3. Ho KKL, Pinsky JL, Kannel WB, Levy D. The epidemiology of heart failure: the Framingham study. J Am Coll Cardiol. 1993;22(4 Suppl A):6A-13A

4. Rosamond W, Flegal K, Furie K, Go A, Greenlund K, Haase N, et al. Heart disease and stroke statistics 2008 update: a report from the American Heart Association Statistics Committee and Stroke Statistics Subcommittee. Circulation. 2008;117(4):25-146.

5. Hunt SA, Baker DW, Chin MH, Cinquegrani MP, Feldman A, Francis GS, et al. ACC/AHA Guidelines for the evaluation and management of chronic heart failure in the adult: executive summary. A report of the American College of Cardiology/ American Heart Association Task Force on Practice Guidelines Committee to revise the 1995 Guidelines for the evaluation and management of heart failure. Circulation. 2001;104(24):2996-3007.

6. Barreto $\mathrm{ACP}$, Drumond Neto C, Mady C, Albuquerque DC, Brindeiro Filho DF, Braile DM, et al. Revisão das II diretrizes da sociedade brasileira de cardiologia para o diagnóstico e tratamento da insuficiência cardíaca. Arq Bras Cardiol. 2002;79(4):1-30.

7. Francis GS. Neurohumoral mechanisms involved in congestive heart failure. Am J Cardiol. 1985;55(2):15A-21A.

8. Cohn JN. Abnormalities of peripheral sympathetic nervous system control in congestive heart failure. Circulation. 1990;82(Suppl I):I-59-I-67.
9. Floras JS. Clinical aspects of sympathetic activation and parasympathetic withdrawal in heart failure. J Am Coll Cardiol. 1993;22(4 Suppl A):72A-84A

10. Gao L, Wang W, Liu Dongmei, Zucker $\mathbb{H}$. Exercise training normalizes sympathetic outflow by central antioxidant mechanisms in rabbits with pacing-induced chronic heart failure. Circulation. 2007;115(24):3095-102.

11. Gao L, Wang W, Li YL, Schultz HD, Liu D, Cornish KG, et al. Superoxid mediates sympathoexcitation in heart failure: roles of angiotensin II and nad(p)h oxidase. Circ Res. 2004;95:937-44.

12. Kimura $Y$, Hirooka $Y$, Sagara $Y$, Ito $K$, Kishi T, Shimokawa $H$, et al. Overexpression of inducible nitric oxide synthase in rostral ventrolateral medulla causes hypertension and sympathoexcitation via an increase in oxidative stress. Circ Res. 2005;96(2):252-60

13. Kishi T, Hirooka Y, Kimura Y, Ito K, Shimokawa H, Takeshita A. Increased reactive oxygen species in rostral ventrolateral medulla contribute to neural mechanisms of hypertension in stroke-prone spontaneously hypertensive rats. Circulation. 2004;109(19):2357-62.

14. Zanzinger J, Czachurski J. Chronic oxidative stress in the RVLM modulates sympathetic control of circulation in pigs. Pflugers Arch. 2000;439(4):489-94.

15. Valenti VE, Sato MA, Fereira C, Abreu LC. Neural regulation of cardiovascular system: brain stem areas. Rev Neurocienc. 2007;15(4):317-20.

16. Cravo SL, Rosa DA, Kalassa F, Korim WS, Hinrichs JM, Ferreira-Neto ML, et al. Os núcleos vasomotores do bulbo e a regulação cardiovascular. Medicina (Ribeirão Preto). 2006;39(1):89-100. 
17. Kaye DM, Lefkovits J, Jennings GL, Bergin P, Broughton A, Esler MD. Adverse consequences of high sympathetic nervous activity in the failing human heart. J Am Coll Cardiol. 1995;26(5):1257-63.

18. Colombari E, Sato MA, Cravo SL, Bergamaschi CT, Júnior RRC, Lopes OU. Role of the medulla oblongata in hypertension. Hypertension. 2001;38/3 Pt 2):549-54.

19. Liu JL, Irvine S, Reid IA, Patel KP, Zucker IH. Chronic exercise reduces sympathetic nerve activity in rabbits with pacing-induced heart failure: a role for angiotensin II. Circulation. 2000;102(15):1854-62.

20. Adams V, Linke A, Krankel N, Erbs S, Gielen S, Mobius-Winkler S, et al. Impact of regular physical activity on the nad(p)h oxidase and angiotensin receptor system in patients with coronary artery disease. Circulation. 2005;111(5):555-62.

21. Adler V, Yin Z, Tew KD, Ronai Z. Role of redox potential and reactive oxygen species in stress signaling. Oncogene. 1999;18(45):6104-11.

22. Rhee SG, Chang TS, Bae YS, Lee SR, Kang SW. Cellular regulation by hydrogen peroxide. J Am Soc Nephrol. 2003;14(8 Suppl 3):S211-5.

23. Ferreira ALA, Matsubara LS. Radicais Livres: conceitos, doenças relacionadas, sistema de defesa e estresse oxidativo. Rev Ass Med Brasil. 1997;43(1):61-8.

24. Kohen R, Nyska A. Oxidation of biological systems: oxidative stress phenomena antioxidants redox reactions and methods for their quantification. Toxicol Pathol. 2002;30(6):620-50.

25. Frantseva MV, Perez Velazquez JL, Carlen PL. Changes in membrane and synaptic properties of thalamocortical circuitry caused by hydrogen peroxide. J Neurophysiol. 1998;80(3):1317-26.

26. Pellmar T. Electrophysiological correlates of peroxide damage in guinea pig hippocampus in vitro. Brain Res. 1986;364(2):377-81.

27. Cardoso LM, Colombari DAS, Menani JV, Chianca Júnior DA, Colombari E. Cardiovascular responses produced by central injection of hydrogen peroxide in conscious rats. Brain Research Bulletin. 2006;71(1-3):37-44.

28. Batlouni M. Insuficiência Cardíaca: da fisiopatologia ao tratamento. Arq Bras Cardiol. 1991;57(1):63-73.

29. Lage SHG, Kopel L, Carvalho MM. Fisiopatologia da insuficiência cardíaca. In: Sousa AGMR, Mansur AJ. SOCESP Cardiologia, $2^{\circ}$ volume. São Paulo: Atheneu, 1996. p. 34-41.

30. Kaye DM, Lefkovits J, Jennings LG, Bergin P, Broughton A, Esler MD. Adverse consequences of high sympathetic nervous activity in the failing human heart. JACC. 1995;26(5)1257-63.

31. Keith M, Geranmayegan A, Sole MJ, Kurian R, Robinson A, Omran AS, et al. Increased oxidative stress in patients with congestive heart failure. J Am Coll Cardiol. 1998;31(6):1352-6.

32. Gao L, Wang W, Li YL, Schultz HD, Liu D, Cornish KG, et al. Superoxido mediates sympathoexcitation in heart failure: roles of angiotensin II and nad(p)h oxidase. Circ Res. 2004;95:937-44.
33. La Rovere MT, Bigger JT, Marcus Fl, Mortara A, Schwartz PJ. Baroreflex sensitivity and heart rate variability in prediction of total cardiac mortality after myocardial infarction. Lancet. 1998;351(9101):478-84.

34. Mortara A, La Rovere MT, Pinna GD, Prpa A, Maestri R, Febo 0, et al. Arterial baroreflex modulation of heart rate in chronic heart failure, clinical and hemodynamic correlates and prognostic implications. Circulation. 1997;96(10):3450-8.

35. Krieger EM, Brum PC, Negrão CE. State-of-the-art lecture: Influence of exercise training on neurogenic control of blood pressure in spontaneously hypertensive rats. Hypertension. 1999;34(4 Pt 2):720-3

36. Véras-Silva AS, Mattos KC, Gava NS, Brum PC, Negrão CE, Krieger EM. Low-intensity exercise training decreases cardiac output and hypertension in spontaneously hypertensive rats. Am J Physiol. 1997;273(6 Pt 2):H2627-31.

37. Bertagnolli M, Camposa C, Schenkela PC, Oliveira VLL, Angelis K, Belló -Klein $A$, et al. Baroreflex sensitivity improvement is associated with decreased oxidative stress in trained spontaneously hypertensive rat. Journal of Hypertension. 2006;24(12):2437-43.

38. Tsutsui H, Ide T, Hayashidani S, Suematsu N, Shiomi T, Wen J, et al. Enhanced generation of reactive oxygen species in the limb skeletal muscles from a murine infarct model of heart failure. Circulation. 2001;104(2):134-6.

39. Hambrecht R, Adams V, Gielen S, Linke A, Mobius-Winkler S, Yu J, et al. Exercise intolerance in patients with chronic heart failure and increased expression of inducible nitric oxide synthase in the skeletal muscle. J Am Coll Cardiol. 1999;33(1):174-9.

40. Heymes C, Bendall JK, Ratajczak P, Cave AC, Samuel JL, Hasenfuss G, et al. Increased myocardial nad(p)h oxidase activity in human heart failure. J Am Coll Cardiol. 2003;41(12):2164-71.

41. Doehner W, Schoene N, Rauchhaus M, Lyva-Leon F, Pavitt DV, Reaveley DA, et al. Effects of xanthine oxidase inhibition with allopurinol on endothelial function and peripheral blood flow in hyperuricemic patients with chronic heart failure: results from 2 placebo-controlled studies. Circulation. 2002;105(22):2619-24.

42. Dixon LJ, Morgan DR, Hughes AM, McGrath LT, El-Sherbeeny NA, Plumb $\mathrm{RD}$, et al. Functional consequences of endothelial nitric oxide synthase uncoupling in congestive cardiac failure. Circulation. 2003;107(13):1725-8.

43. Mann DL, Reid MB. Exercise training and skeletal muscle inflammation in chronic heart failure: feeling better about fatigue. J Am Coll Cardiol. 2003;42(5):869-72.

44. Adams V, Nehrhoff B, Spate U, Linke A, Schulze PC, Baur A, et al. Induction of iNOS expression in skeletal muscle by IL-1 $\beta$ and NFKB activation: an in vitro and in vivo study. Cardiovasc Res. 2002;54:95-104.

45. Adams V, Jiang H, Yu J, Mobius-Winkler S, Fiehn E, Linke A, et al. Apoptosis in skeletal myocytes of patients with chronic heart failure is associated with exercise intolerance. J Am Coll Cardiol. 1999;33(4):959-65.

46. Harrington D, Ankers SD, Chua TP, Webb-People KM, Ponikowski PP, PooleWilson PA, et al. Skeletal muscle function and its relation to exercise tolerance in chronic heart failure. J Am Coll Cardiol. 1997;30(7):1758-64. 
47. Linke A, Adams V, Schulze PC, Erbs S, Gielen S, Fiehn E, et al. Antioxidante effects of exercise training in patients with chronic heart failure: Increase radical scavenger enzyme activity in skeletal muscle. Circulation. 2005;111(14):1763-70.

48. Ennezat PV, Malendowicz SL, Testa M, Colombo PC, Cohen-Solal A, Evans $T$, et al. Physical training in patients with chronic heart failure enhances the expression of genes encoding antioxidative enzymes. J Am Coll Cardiol. 2001;38(1):194-8.

49. Francis GS. The relationship of the sympathetic nervous system and the reninangiotensin system in congestive heart failure. Am Heart J. 1989;118(3):642-8.

50. Francis GS, Cohn JN, Johnson G, Rector TS, Goldman S, Simon A. Plasma norepinephrine, plasma renin activity, and congestive heart failure. Relations to survival and the effects of therapy in V-HeFT II. The V-HeFT VA Cooperative Studies Group. Circulation. 1993;87(6 Suppl):VI40-8.

51. Kinugawa T, Ogino K, Kitamura H, Saitoh M, Omodani H, Osaki S, et al. Catecholamines, renin-angiotensin-aldosterone system, and atrial natriuretic peptide at rest and during submaximal exercise in patients with congestive heart failure. Am J Med Sci. 1996;312(3):110-7.

52. Reid IA. Interactions between Ang II, sympathetic nervous system, and baroreceptor reflexes in regulation of blood pressure. Am J Physiol. 1992;262(6 Pt 1):E763-78.

53. Liu JL, Murakami H, Sanderford M, Bishop VS, Zucker IH. Ang II and baroreflex function in rabbits with CHF and lesions of the area postrema. Am J Physiol. 1999;277(1 Pt 2):H342-50.

54. DiBona GF, Jones SY, Brooks VL. Ang II receptor blockade and arterial baroreflex regulation of renal nerve activity in cardiac failure. Am J Physiol. 1995;269(5 Pt 2):R1189-96.

55. Rueckschloss U, Quinn MT, Holtz J, Morawietz H. Dose-dependent regulation of nad(p)h oxidase expression by angiotensin II in human endothelial cells: protective effect of angiotensin II type 1 receptor blockade in patients with coronary artery disease. Arterioscler Thromb Vasc Biol. 2002;22(11):1845-51.

56. Mollnau H, Wendt M, Szocs K, Lassegue B, Schulz E, Oelze M, et al. Effects of angiotensin II infusion on the expression and function of nad(p)h oxidase and components of nitric oxide/cGMP signaling. Circ Res. 2002;90(4):E58-65.
57. Lassenge B, Clempus RE. Vascular nad(p)h oxidases: specific features, expression, and regulation. Am J Physiol. 2003;285(2):R277-97.

58. Brooks VL, Osborn JW. Hormonal-sympathetic interactions in long-term regulation of arterial pressure: an hypothesis. Am J Physiol. 1995;268(6 Pt 2):R1343-58.

59. Grassi G, Seravalle G, Cattaneo BM, Lanfranchi A, Vailati S, Giannattasio C, et al. Sympathetic activation and loss of reflex sympathetic control in mild congestive heart failure. Circulation. 1995;92(11):3206-11.

60. Luchner A, Stevens TL, Borgeson DD, Redfield MM, Bailey JE, Sandberg SM, et al. Angiotensin II in the evolution of experimental heart failure. Hypertension. 1996;28(3):472-7.

61. Liu JL, Irvine S, Reid IA, Patel KP, Zucker IH. Chronic exercise reduces sympathetic nerve activity in rabbits with pacing-induced heart failure: a role for angiotensin II. Circulation. 2000;102(15):1854-62.

62. Georgiou D, Chen Y, Appadoo S, Belardinelli R, Greene R, Parides MK, et al. Cost-effectiveness analysis of long-term moderate exercise training in chronic heart failure. Am J Cardiol. 2001;87(8):984-8; A4.

63. Belardinelli R, Georgiou D, Cianci G, Purcaro A. Exercise training for patients with chronic heart failure reduced mortality and cardiac events and improved quality of life. West J Med. 2000;172(1):28.

64. Roveda F, Middlekauff HR, Rondon MU, Reis SF, Souza M, Nastari L, et al. The effects of exercise training on sympathetic neural activation in advanced heart failure: a randomized controlled trial. J Am Coll Cardiol. 2003:42(5):854-60.

65. Larsen Al, Dickstein K. Exercise training in congestive heart failure: a review of the current status. Minerva Cardioangiol. 2005;53(4):275-86.

67. Rontoyanni VG, Chowienczyk PJ, Sanders TA. Postprandial lipaemia does not affect resting haemodynamic responses but does influence cardiovascular reactivity to dynamic exercise. Br J Nutr. 2010;27:1-9.

68. Houston MC. The role of cellular micronutrient analysis, nutraceuticals, vitamins, antioxidants and minerals in the prevention and treatment of hypertension and cardiovascular disease. Ther Adv Cardiovasc Dis. 2010;4(3):165-83 